\title{
Technique of Percutaneous Transforaminal Endoscopic Discectomy for the Treatment of Lumbar Disc Herniation
}

\author{
Rama Shankar Gupta, Xiao-Tao Wu*, Xin Hong, Arjun Sinkemani \\ Department of Spinal Surgery, Southeast University School of Medicine Affiliated Zhongda Hospital, Nanjing, \\ China \\ Email: avinov5@gmail.com, Rama2007@hotmail.com,
}

Received 16 June 2015; accepted 27 July 2015; published 30 July 2015

Copyright (C) 2015 by authors and Scientific Research Publishing Inc.

This work is licensed under the Creative Commons Attribution International License (CC BY). http://creativecommons.org/licenses/by/4.0/

(c) (i) Open Access

\section{Abstract}

Percutaneous Transforaminal Endoscopic Discectomy is a minimally invasive surgery with little pain, less blood loss, less hospital stay and the surgery can be done in local anesthesia, which was started during late $20^{\text {th }}$ century. Kambin and Gellmann in 1973 in the United States and Hijikata in Japanin 1977 individually preformed posterolateral percutaneous nucleotomy for the resection of the nucleus pulposus and release of compressed exiting nerve root, which is now spreading through the world and many surgeons are developing their skill but it needs experience and patience for successful outcomes. Along with advanced instruments now the surgery can be performed only giving a small skin incision of $8-10 \mathrm{~mm}$ and is as effective as the conventional method of surgery and open microdiscectomy surgery for the treatment of symptomatic lumbar disc herniation. In this review, we are explaining the technique of minimally invasive Percutaneous Transforaminal Endoscopic Discectomy surgery along its advantages and complications which can be encountered while performing this technique.

\section{Keywords}

Lumbar Disc Herniation, Herniated Nucleus Pulposus, Percutaneous Transforaminal Endoscopic Discectomy, Nucleotomy, Root Injury, Safety Triangle

\section{Introduction and History}

A spinal disc herniation [1] is a medical condition affecting the spine in which a tear in the outer fibrous ring

\footnotetext{
"Corresponding author.
}

How to cite this paper: Gupta, R.S., Wu, X.-T., Hong, X. and Sinkemani, A. (2015) Technique of Percutaneous Transforaminal Endoscopic Discectomy for the Treatment of Lumbar Disc Herniation. Open Journal of Orthopedics, 5, 208-216. 
(annulus fibrosus) of an intervertebral disc allows the soft, central portion (nucleus pulposus) to bulge out beyond the damaged outer rings of annulus firbosus, causing mild to severe pain, paresthesia \& paralysis of the limbs. Lumbar discherniation (LDH) is a common orthopedic disease which causes medical and economic burdens to families, society and country [2]. Figure 1 shows the bulging of nucleus pulposus and compression of the nerve which causes lower back pain and Figure 2 is a typical magnetic resonance imaging (MRI) of a herniated nucleus pulposus (HNP). Most patients with lumbar disc herniation get better with conservative treatment but some groups of people need surgery to get relief from pain and other symptoms.

Surgery for lumbar disc herniation can be divided into two main groups: Conventional (Traditional or open) versus minimally invasive and posterior approach versus posterolateral approach. The conventional method for treating LDH includes laminotomy, flavectomy, and dissection of the HNP fragment. Mixter and Barr in 1934 [3] for the first time they introduced the treatment of lumbar disc herniation surgically by performing an open laminectomy and discectomy which was also known as love's technique. In late $20^{\text {th }}$ century, some surgeons begin to treat the LDH by minimally invasive procedure by the introduction of different angled microscope. Caspar and Yasargil were the two authors who changed the open laminectomy into open microdiscectomy [4]. Foley KT, Smith MM [5] and Destandeau [6] developed the technique to access the HNP through the interlaminar space i.e. via the interlaminar approach, by further development in the utilization of a spinal endoscope and tubular

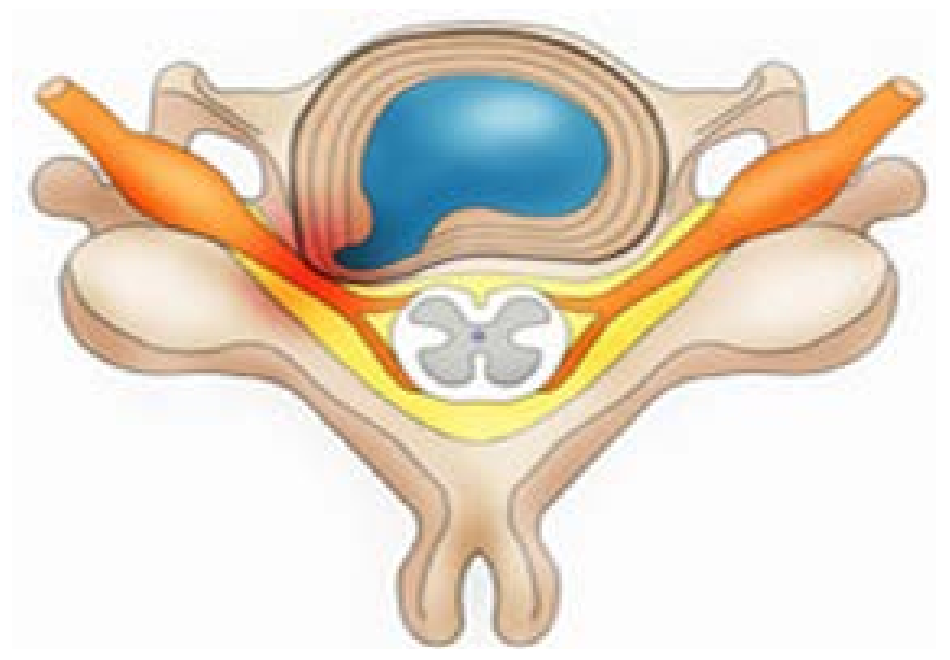

Figure 1. A schematic illustration of herniated disc and compression of exiting nerve root. Figure taken from google.

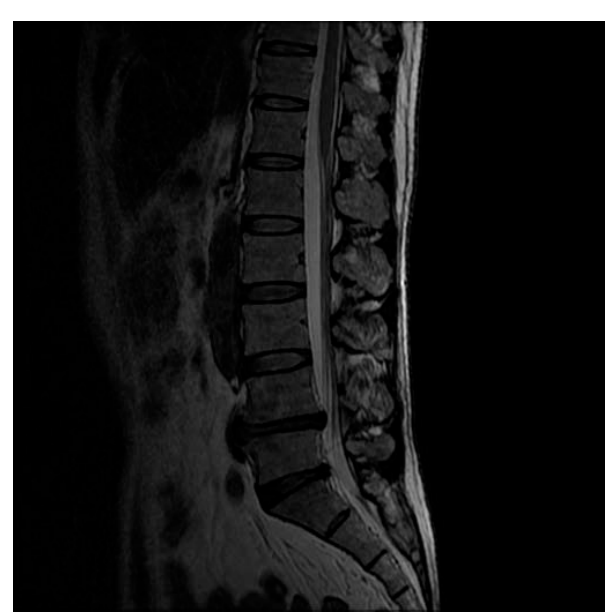

(a)

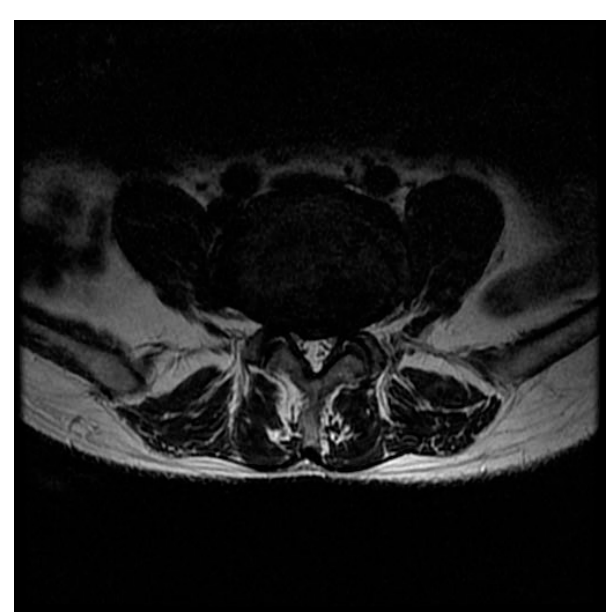

(b)

Figure 2. (a) Magnetic resonance imaging (MRI) of a herniated nucleus pulposus (HPN) at L4/5 and L5/S1 level. (b) Shows the compression of nerve. (Images taken from Zhongda Hospital). 
retractor, which in recent days is known as microendoscopic discectomy (MED). In MED, a microscope is used and the HNP is approached through the posterior direction instead of transforaminal approach. Kambin and Gellmann in 1973 [7] in the united states and Hijikata in Japan in 1977 [8] individually preformed the posterolateral percutaneous nucleotomy for resection of the nucleus pulposus and release of compressed nerve root without direct visualization and later in 1983, Fost and Housman reported the direct visualization of the intervertebral disc space with a modified arthroscope which is now known as percutaneous endoscopic discectomy (PED) or percutaneous transforaminal endoscopic discectomy (PTED) [9]. Thanks to their efforts for developing the spinal instruments for endoscopic lumbar surgery through foraminal space. Percutaneous endoscopic discectomy has been established as a minimally invasive surgery and is spreading throughout the world. Many other authors also have developed new techniques such as "percutaneous discoscopy" a biportal endoscopic posterolateral technique with modified instruments for direct view, current single portal endoscopic discectomy was developed by Yeung et al. [10]-[12]. Mayer introduced the concept of percutaneous endoscopic laser discectomy combining forceps and laser for the treatment of LDH. Nowadays minimally invasive surgery has gained the attention of many surgeons and has become a subject of growing interest. The aim of minimally invasive technique is to provide less trauma and minimal postoperative pain to the patient of lumbar disc herniation without producing iatrogenic morbidity, which is usually associated with open surgical techniques. Eventhough this technique (PTED or PED) is getting more and more popular and is gradually increasing the number of surgeons but still now there are only few active PED surgeons, because to perform this surgery it needs many years of training and patience. This technique is purely used to treat the diseases of lumbar region disc herniation at different level and in this review article we are going to know about the technique of percutaneous transforaminal endoscopic discectomy in the treat of lumbar disc herniation (LDH).

\section{Surgical Technique}

For the treatment of lumbar disc herniation surgically, patient are selected according to the result of their radiological investigations such as magnetic resonance imaging (MRI), computed tomography (CT), physical examination, suitable for local anesthesia and failure of conservative treatment. The patient is kept in prone position on a standard radiologically compatible spine table and well-padded at the pressure points (Figure 3). All the instruments are kept ready along with C-arm image intensifier. Careful documentation of the admitting lumbar site is taken and the cannula insertion point is determined before surgery from the CT and MRI. The procedure can be done under local anesthesia or intravenous sedation but most surgeons like to do under local anesthesia. After preparing and draping the site for operative procedure according to the hospital's usual sterile fashion the

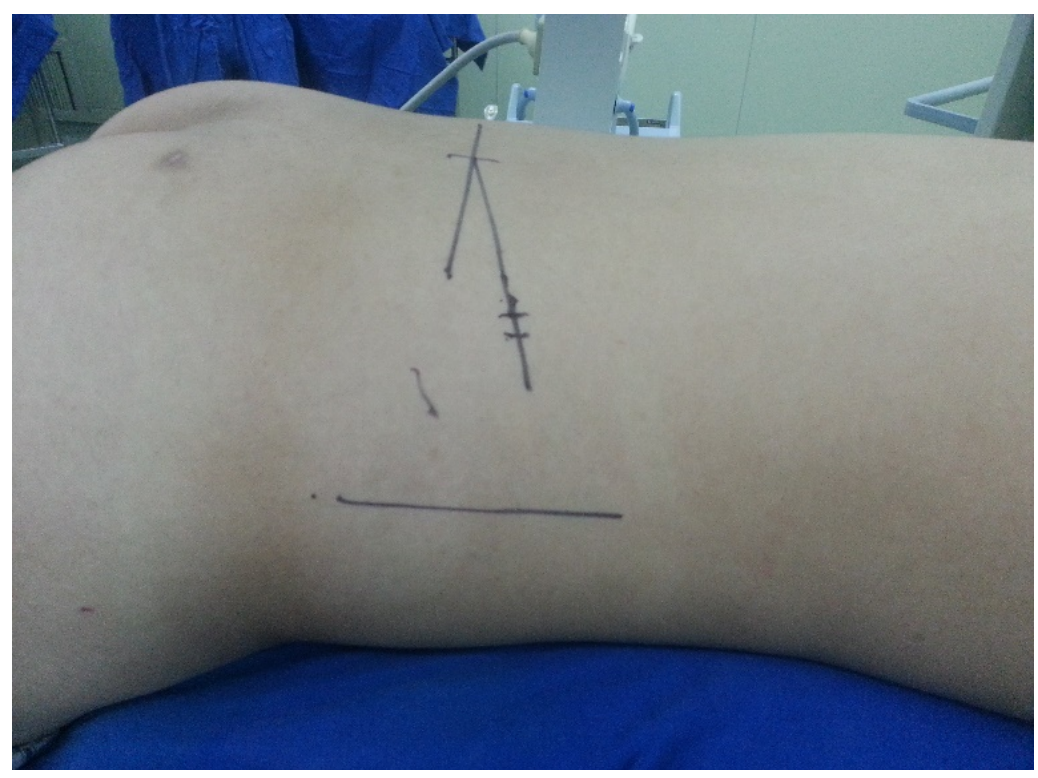

Figure 3. Showing the position of the patient and marking the point where the incision is given to induce the canula. Photograph taken from Zhongda Hospital. 
surgeon gives the local anesthesia, mostly $1 \%$ lidocaine is given at the distance of 8 to $12 \mathrm{~cm}$ from the midline (Figure 4), although the distance varies from patient to patient but in general pattern it is about $8 \mathrm{~cm}$ in thin patient and greater in fat patients from the midline. For this procedure it requires about 10 to $15 \mathrm{ml}$ of lidocaine. After giving the local anesthesia the surgeon uses a $25 \mathrm{~cm} 18$ gauge needle to advance and place it in the disc space through safety triangle or Kambin's triangle (Figure 5).

The insertion of the needle is by walking technique which prevents injuring the nerve root coming out from the cranial side of the intervertebral foramen (Figure 5), the walking technique is performed under the visualization taken by C-arm image intensifier in two plane anterior-posterior and lateral view, the puncture needle is firstly kept on the caudal pedicle and then using the walking technique, the needle is safely inserted into the intervertebral disc. Next step after insertion of the needle at the proper site is to dye the nucleus pulposus and the herniated mass by using indigo carmine, after dyeing the nucleus pulposus changes to blue color but the annulus

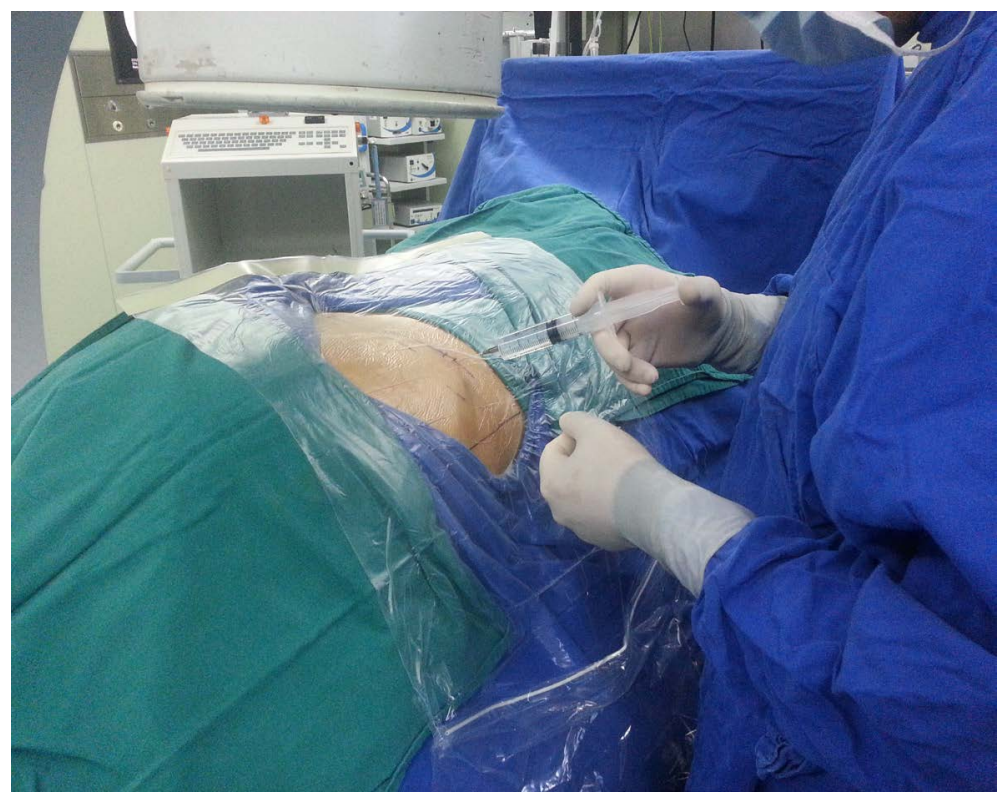

Figure 4. showing the distance from midline where the anesthesia is given which is about $12 \mathrm{~cm}$ in this patient. Photograph taken from Zhongda Hospital.

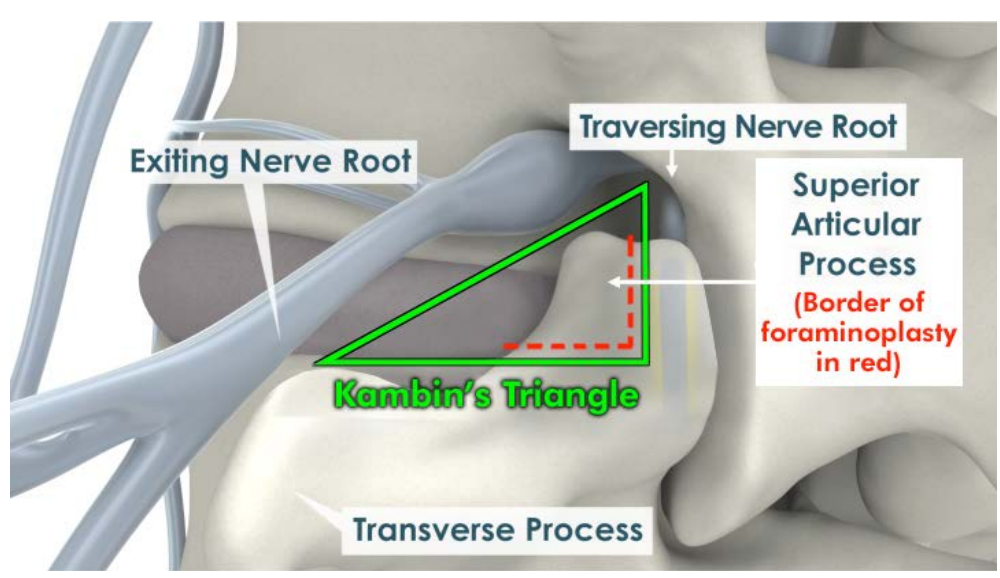

Figure 5. Schematic diagram showing Exiting nerve root, transverse process of the vertebral body and traversing nerve root which forms the safety triangle or Kambin's triangle. It is also showing the articular part of the facet which is used to dissect in foraminoplasty. Figure taken from international journal of spine surgery. 
fibrosus stays white because it doesn't stain with the dye but the epidural space is red due to the presence of vessels (Figure 6(a), Figure 6(b)).

The difference in the color allows the surgeon to easily identify the herniated mass and to safely remove it. After this, a guide pin is inserted through the puncture needle into the intervertebral disc and then the obturater and the cannula are inserted after giving an 8 to $10 \mathrm{~mm}$ of skin incision (Figure 7). The herniated mass is removed by inside-out technique and hand down technique after the insertion of the cannula through the interforaminal space. To know the complete removal of the herniated mass the cannula is moved towards the epidural space and the pulsation if the Dural tube is felt, which is a confirmatory indication of decompression. After the removal of the herniated nucleus pulposus and decompressing the nerve, all the instruments are removed carefully, if there is bleeding at the insertion point then it is stopped by pressing on it for few minutes and then a single or two skin stiches are given at the incision point (Figure 8). A sterile dressing is use to cover the stich site.

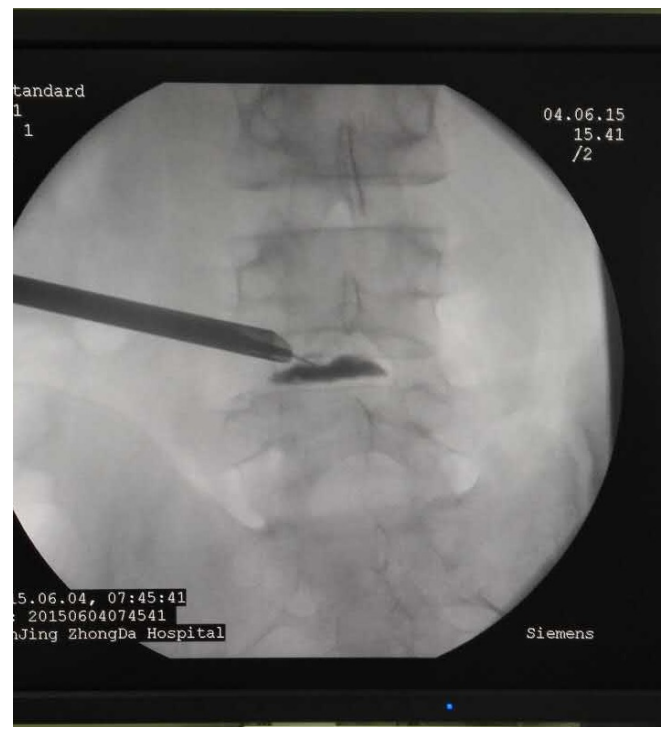

(a)

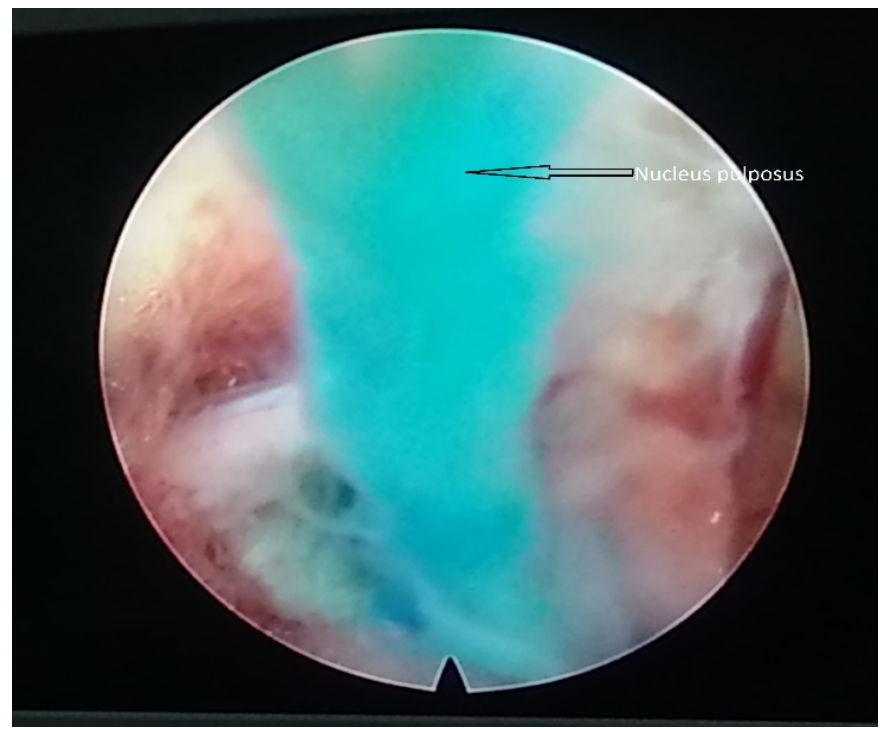

(b)

Figure 6. (a) and (b) shows the dying of nucleus pulposus and shows the color of the nucleus pulposus and its surrounding structures Photograph taken from Zhongda Hospital.

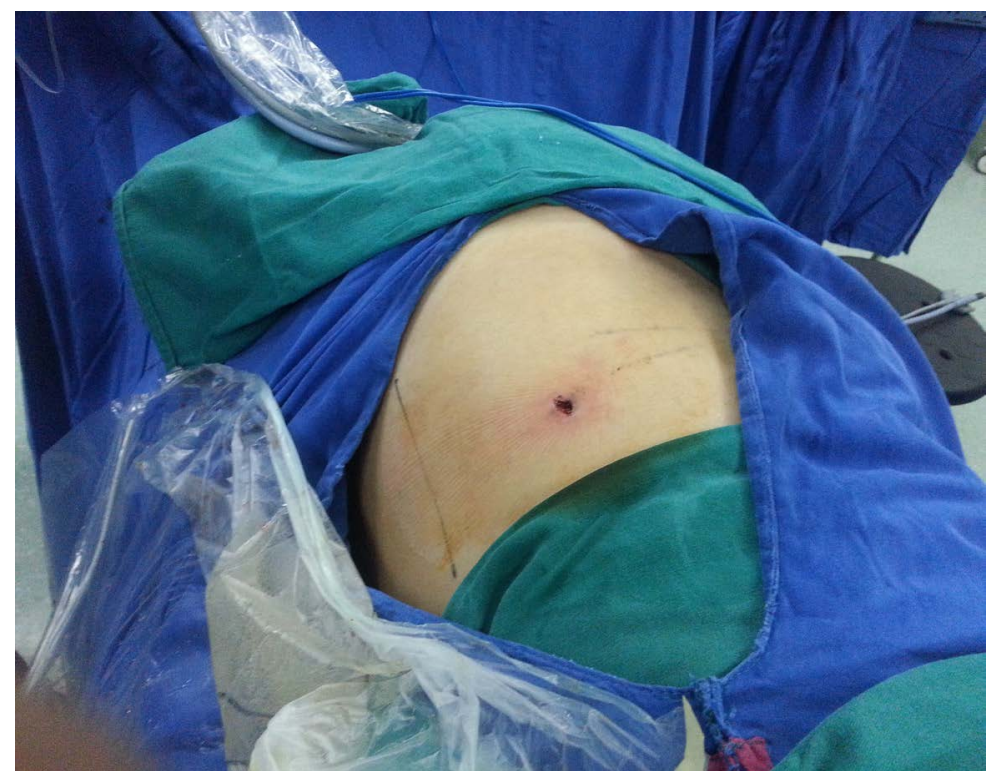

Figure 7. Shows the incision length. Photograph taken from Zhongda Hospital. 


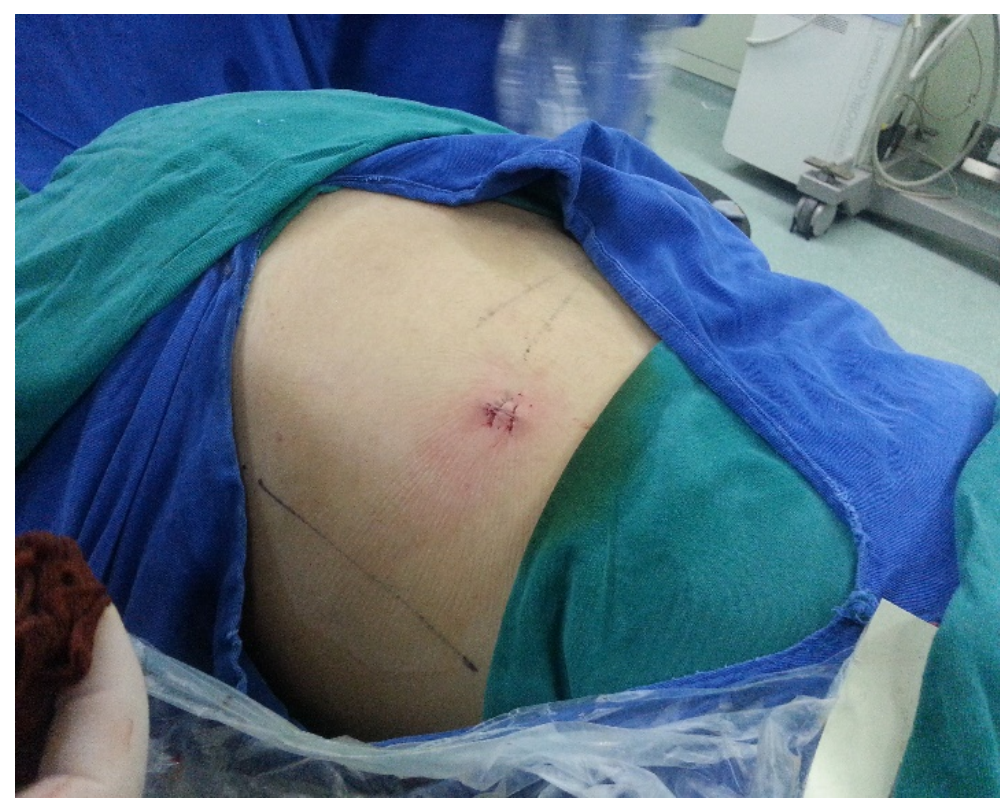

Figure 8. Shows the stiches given after the operation. Photograph taken from Zhongda Hospital.

\section{Post Surgical Schedule}

The patient can start walking and standing after 1 - 2 hours, if one has no feeling of numbness but if he feels numbness then rest is mandatory. Some patients' feels numbness after the surgery and it is due to the infiltration of the local anesthesia (lidocaine) in the exiting nerve. Nowadays, in some hospitals this surgery is done as an OPD procedure and the patient is discharged on the same day but usually the patients are discharged 1 to 3 days after surgery. Patient is allowed to work in their office or to do minor works 4 - 5 days after surgery and heavy works such as lifting things 6 - 8 weeks later from the day of surgery to prevent the recurrence of disc herniation. Sports activity is also allowed after 6 to 8 weeks later. Patients were given antibiotics 1 to 3 days post-operatively to prevent infections and pain relieving medication to prevent from post-operative pain.

\section{Surgical Indication}

Intracanalherniated nucleus pulposus (HNP) at L1/2 to L4/5 without migration and with migration:

HNP without migration is a good indication for PTED but migrated HNP also can be treated by PTED using additional technique known as Pediculotomy, which is a technically demanding surgery and is able to remove the upward and downward migrated HNP [13]. For more hidden migrated HNP Dezawa et al. introduced the translaminar surgical procedure which is done along with PED [14].

IntracanalHNP at L5/S1 level with a high iliac crest:

As reported by Yeung and Tsou [11] most of their cases (50\%) were operated at the L5/S1 level, which is known as "high iliac" cases, in which the iliac crest hinders the insertion of the cannula in the intervertebral disc through interforaminal space that is why it is not possible to operate at that level according to the standard technique. For this problem Lee et al. [15] introduced a new technique known as froaminoplastic approach, which allows the insertion of the cannula in the intervertebral space by enlarging the intervertebral foramen by foraminoplasty.

Far-lateral HNP:

For far lateral HNP the cannula can be directly inserted on it and can be easily removed under local anesthesia, there is no need to insert the cannula through the interforaminal space. Far lateral HNP can be removed at any level through posterolateral approach [16] [17].

Recurrent HNP:

Recurrent HNP is very difficult to perform surgery through love's method or micro love's method due tissue scarring and adhesion of the nearby tissues to the herniated mass and it is a demanding technique to perform in 
such cases but by PED technique it is a safe and easy to perform. Ruetten et al. stated the usefulness of PED on recurrent HNP [18] and emphasized its utility [19]. Shin et al. [20] did revision surgeries on 41 cases and found excellent outcomes in more than $90 \%$ cases.

\section{Clinical Outcome}

Yeung and Tsou [10] reported that the clinical outcome of PTED in comparison with conventional method of treatment for lumbar disc herniation was almost similar by performing PTED in 307 cases, in which they found that the outcome was satisfactory in $90 \%$ of cases which is also observed in conventional method. Another author named Ruetten et al. [21] also have conducted PTED in several patients and compared it with microdiscectomy where he found that $82 \%$ of patients have no pain after surgery and few have occasional pain those who were treated by microdiscectomy. Birkenmaier et al. [22] explained why present technique of surgery PTED is safe and more beneficial than the conventional technique of surgery after comparing the studies of both technique in similar numbers and concluded that current technique of PTED is beneficial than conventional technique due to shorter operating time, less blood loss, less surgery related complications, less surgical site pain after surgery, less need of postoperative medication for pain, shorter hospital stay, return to work in less time and also due to cosmetic and economic point of view.

\section{Complications}

Regarding complication for the treatment of lumbar disc herniation by percutaneous transforaminal endoscopic discectomy, it has less complication than other techniques which is an advantage for this technique because of less tissue injury, small incision and minimal internal tissue damage along with minimized scar tissue [23] [24], even though it have few complications such as dysaesthesia or hyperaesthesia. Injury to the exiting nerve also occurs due to faulty insertion of the cannula and over infiltration of local anesthesia lidocaine at the operation level, exiting nerve root injury can be prevented by carefully insertion of cannula by walking technique and intermittent fluoroscopic view, and giving adequate amount of lidocaine prevents nerve irritation (exiting nerve and dorsal root ganglion). Cauda equine injury, Dural tear, Hematoma formation, Surgical site infection and Major vessel injury are some other complications which can occur during the percutaneous endoscopic discectomy. To prevent this kind of complications surgeon should be well trained and have patience, as Transforaminal Endoscopic Discectomy Surgery has a steep learning curve which requires many years of training and experience, patients who were treated at the beginning of the learning curve have bad experience of pain and outcome was worst [25]-[29]. Another most important complication while performing TED is intracranial hypertension which surgeon should be careful of it and the indication of intracranial hypertension is headache, seizure and neck pain during the surgery, so the surgeon should always ask the patient about it intermittently during the operation and try to finish the operation as soon as possible without complications. Choi et al. [30] reported that some of his patients have seizure and neck pain during the operation which is a warning sign because it may be fatal and cause of death due to intracranial hypertension.

\section{Future Research}

To know the effectiveness of the PTED for the treatment of lumbar disc herniation a high-quality, randomized controlled trial with large sample size should be done by comparing with conventional discectomy, open microdiscectomy and other techniques. Recently two kinds of new techniques are introduced using PED to treat disc herniation; lumbar spinal canal stenosis by laminectomy with a high speed drill [31] and radiofrequency thermal annuloplasty for discogenic low back pain [32] [33].

\section{Conclusion}

This review assessed the technique and effectiveness of Percutaneous Transforaminal Endoscopic Discectomy for the treatment of symptomatic Lumbar Disc Herniation, it also emphasized its advantages over other methods of discectomy and conventional method of treatment. There are several authors who have published their papers to show the different techniques of PTED and its usefulness, but there are not so many trials regarding this technique which can definitely state the effectiveness. Eventhough as far as the article is published, it shows that PTED is safe and as effective as the other techniques. This technique also has some drawbacks, such as surgeons 
need long learning time and experience with patience.

\section{References}

[1] American English: Slipped Disk.

[2] Dagenais, S., Caro, J. and Haldeman, S. (2008) A Systematic Review of Lowback Pain Cost of Illness Studies in the United States and Internationally. The Spine Journal, 8, 8-20. http://dx.doi.org/10.1016/j.spinee.2007.10.005

[3] Mixter, W. and Barr, J. (1934) Rupture of the Intervertebral Disc with Involvement of the Spinal Canal. The New England Journal of Medicine, 211, 210-215. http://dx.doi.org/10.1056/NEJM193408022110506

[4] Caspar, W. (1977) A New Surgical Procedure for Lumbar Disk Herniation Causing Less Tssue Damage through a Microsurgical Approach. Advances in Neurosurgery, 4, 74-77.

[5] Foley, K.T. and Smith, M.M. (1997) Microendoscopic Approach to Far-Lateral Lumbar Disc Herniation. Techniques in Neurosurgery, 3, 301-307.

[6] Ddestandeau, J. (2004) Technique Features of Endoscopic Surgery for Lumbar Disc Herniation: 191 Patients. Neurochirurgie, 50, 6-10.

[7] Kambin, P. and Gellman, H. (1983) Percutaneous Lateral Discectomy of the Lumbar Spine: A Preliminary Report. Clinical Orthopaedics and Related Research, 174, 127-132. http://dx.doi.org/10.1097/00003086-198304000-00015

[8] Hijikata, S. (1989) Percutaneous Nucleotomy. A New Concept Techinque and 12 Years' Experience. Clinical Orthopaedics and Related Research, 238, 9-23.

[9] Forst, R. and Hausmann, B. (1983) Nucleoscopy-A New Examination Technique. Archives of Orthopaedic and Trauma Surgery, 101, 219-221. http://dx.doi.org/10.1007/BF00436774

[10] Yeung, A.T. (2000) The Evolution of Percutaneous Spinal Endoscopy and Discectomy: State of the Art. Mount Sinai Journal of Medicine, 67, 327-332.

[11] Yeung, A.T. and Tsou, P.M. (2002) Posterolateral Endoscopic Excision for Lumbar Disc Herniation: Surgical Technique, Outcome, and Complications in 307 Consecutive Cases. Spine, 27, 722-731.

[12] Yeung, A.T. and Yeung, C.A. (2007) Minimally Incasive Technique for the Management of Lumbar Disc Herniation. Orthopedic Clinics of North America, 38, 363-372. http://dx.doi.org/10.1016/j.ocl.2007.04.005

[13] Lee, S., Kim, S.K., Lee, S.H., Kim, W.J., Choi, W.C., Choi, G. and Shin, S.W. (2007) Percutaneous Endoscopic Lumbar Discectomy for Migrated Disc Herniation: Classification of Disc Migration and Surgical Approaches. European Spine Journal, 16, 431-437. http://dx.doi.org/10.1007/s00586-006-0219-4

[14] Dezawa, A., Mikami, H. and Sairyo, K. (2011) Percutaneous Endoscopic Translaminar Approach for Herniated Nucleus Pulposus in the Hidden Zone of the Lumbar Spine. Asian Journal of Endoscopic Surgery, 5, 200-203.

[15] Lee, S.H., Kang, H.S., Choi, G., Kong, B.J., Ahn, Y., Kim, J.S. and Lee, H.Y. (2010) Foraminoplastic Ventral Epidural Approach for Removal of Extruded Herniated Fragment at the L5-S1 Level. Neurologia Medico-Chirurgica (Tokyo), 50, 1074-1078. http://dx.doi.org/10.2176/nmc.50.1074

[16] Sasani, M., Ozer, A.F., Oktenoglu, T., Canbulat, N. and Sarioglu, A.C. (2007) Percutaneous Endoscopic Discectomy for Far Lateral Lumbar Disc Herniations: Prospective Study and Outcome of 66 Patients. Minimally Invasive Neurosurgery, 50, 91-97. http://dx.doi.org/10.1055/s-2007-984383

[17] Kitagawa, Y., Sairyo, K., Shibuya, I., Kitahama, Y., Kanamori, Y., Koga, S., Matsumoto, H., Sumita, T., Yamada, A. and Dezawa, A. (2012) Minimally Invasive and Simultaneous Removal of Herniated Intracanal and Extracanal Lumbar Nucleus Pulposus with a Percutaneous Spianal Endoscope. Asian Journal of Endoscopic Surgery, 5, 183-186. http://dx.doi.org/10.1111/j.1758-5910.2012.00144.x

[18] Rutten, S., Komp, M. and Godolias, G. (2005) An Extreme Lateral Access for the Surgery of Lumbar Disc Herniation Inside the Spinal Canal Using the Full-Endoscopic Uniportal Transforaminal Approach-Technique and Prospective Results of 463 Patients. Spine, 30, 2570-2578. http://dx.doi.org/10.1097/01.brs.0000186327.21435.cC

[19] Rutten, S., Komp, M., Merk, H. and Godolias, G. (2009) Recurrent Lumbar Disc Herniation after Conventional Discectomy: A Prospective, Randomized Study Comparing Full-Endoscopic Interlaminar and Transforaminal versus Microsurgical Revision. Journal of Spinal Disorders \& Techniques, 22, 122-129. http://dx.doi.org/10.1097/BSD.0b013e318175ddb4

[20] Shin, K.H., Chang, H.G., Rhee, N.K. and Lim, K.S. (2011) Revisional Percutaneous Full Endoscopic Disc Surgery for Recurrent Herniation of Previous Open Lumbar Discectomy. Asian Spine Journal, 5, 1-9. http://dx.doi.org/10.4184/asj.2011.5.1.1

[21] Rutten, S., Komp, M., Merk, H. and Godolias, G. (2008) Full-Endoscopic Interlaminar and Transforaminal Lumbar Discectomy versus Conventional Microsurgical Technique: A Prospective, Randomized, Controlled Study. Spine, 33, 


\section{1-939. http://dx.doi.org/10.1097/BRS.0b013e31816c8af7}

[22] Birkenmaier, C., Komp, M., Leu, H.F., Wegener, B. and Rutten, S. (2013) The Current State of Endoscopic Disc Surgery: Review of Controlled Studies Comparing Full-Endoscopic Procedures for Disc Herniations to Standard Procedures. Pain physician, 16, 335-344.

[23] Knight, M., Goswami, A. and Patko, J.T. (1999) Endoscopic Laser Foraminoplasty and an Aware-Stat Surgery: A Treatment Concept and 2-Year Outcome Analyses. Arthoskopie, 12, 62-73. http://dx.doi.org/10.1007/s001420050074

[24] Knight, M.T., Goswami, A., Patko, J.T., et al. (2001) Endoscopic Foraminoplasty: A Prospective Study on 250 Consecutive Patients with Independent Evaluation. Journal of Clinical Laser Medicine \& Surgery, 19, 73-81. http://dx.doi.org/10.1089/104454701750285395

[25] Haag, M. (1999) Transforaminal Endoscopic Microdiscectomy. Indications and Short-Term to Intermediate-Term Results. Orthopade, 28, 615-621.

[26] Kafadar, A., Kahraman, S. and Akboru, M. (2006) Percutaneous Endoscopic Transforaminal Lumbar Discectomy: A critical Appraisal. Minimally Invasive Neurosurgery, 49, 74-79. http://dx.doi.org/10.1055/s-2006-932184

[27] Kim, M.J., Lee, S.H. and Jung, E.S. (2007) Targeted Percutaneous Transforaminal Endoscopic Diskectomy in 295 Patients: Comparison with Results of Microscopic Diskectomy. Surgical Neurology, 68, 623-631. http://dx.doi.org/10.1016/j.surneu.2006.12.051

[28] Shim, Y.B., Lee, N.Y. and Huh, SH. (2007) Endoscopic Spinal Surgery for Herniated Lumbar Discs. Journal of Korean Neurosurgical Society, 41, 241-245. http://dx.doi.org/10.3340/jkns.2007.41.4.241

[29] Tzaan, W.C. (2007) Transforaminal Percutaneous Endoscopic Lumbar Discectomy. Chang Gung Medical Journal, 30, 226-234.

[30] Choi, G., Kang, H.Y., Modi, H.N., Prada, N., Nicolau, R.J., Joh, J.Y., Pan, W.J. and Lee, S.H. (2011) Risk of Developing Seizure after Percutaneous Endoscopic Lumbar Discectomy. Journal of Spinal Disorders \& Techniques, 24, 83-92. http://dx.doi.org/10.1097/BSD.0b013e3181ddf124

[31] Ruetten, S., Komp, M., Hahn, P. and Oezdemir, S. (2013) Decompression of Lumbar Lateral Spinal Stenosis: FullEndoscopic, Interlaminar Technique. Operative Orthopädie und Traumatologie, 25, 31-46. http://dx.doi.org/10.1007/s00064-012-0195-2

[32] Sairyo, K., Kitagawa, Y. and Dezawa, A. (2013) Percutaneous Endoscopic Discectomy and Thermalan-Nuloplasty for Professional Athletes. Asian Journal of Endoscopic Surgery, 6, 292-297. http://dx.doi.org/10.1111/ases.12055

[33] Tsou, P.M., Alan Yeung, C. and Yeung, A.T. (2004) Postero-Lateral Transforaminal Selective Endoscopic Discectomy and Thermal Annuloplasty for Chronic Lumbar Discogenic Pain: Aminimal Access Visualized Intradiscal Surgical Procedure. The Spine Journal, 4, 564-573. http://dx.doi.org/10.1016/j.spinee.2004.01.014 